\title{
INTERDISCIPLINARY REMOTE DIDACTIC LABORATORY "G.SAVASTANO": PHYSICS APPLICATION
}

\author{
A. Apergis ${ }^{1)}$, S. Rapuano ${ }^{2)}$ IEEE Member, F. Zoino ${ }^{2,3)}$ \\ ${ }^{1)}$ Physics Department, Aristotle University, Thessaloniki, Greece, E.mail aaperg@physics.auth.gr \\ ${ }^{2)}$ Department of Engineering, University of Sannio, Corso Garibaldi, 107, 82100, Benevento, Italy \\ Ph.: +390824 305817, Fax: +39 0824 305840, E-mail: \{rapuano, frazoino\}@unisannio.it \\ ${ }^{3)}$ Didagroup S.p.A., Viale Dei Sanniti, 1, S.Giorgio del Sannio (BN), Italy
}

\begin{abstract}
E-learning offers to the student's didactic tools and course materials over Internet. By distributing tools and materials in this fashion, students isolated from the University environment can still obtain University degrees in many fields. Courses that include laboratory sessions have traditionally required students to be on-site. This is primarily due to the need for laboratory resources to be located in a single site for both practical and safety reasons. This fact hampered the freedom of non traditional (off campus) students to achieve their educational goals. The web adaptations of software packages such as LabVIEW allow control of experiments over Internet. Remote laboratories then become possible.

In this framework the Remote Laboratory Distributed on Geographical Network LA.DI.RE. "G.Savastano" of University of Sannio [1] was born, with the aim of developing a virtual learning environment to deliver, through the distance learning methodologies, theoretical lessons as well as laboratory activity on real measurement instrumentation.

The paper highlights the interdisciplinary aspect of LA.DI.RE. "G. Savastano", showing the possibility to provide different real experiments independent of software development environment, thanks to use of an open Learning Management System (LMS) and concerning different didactic fields. In this case the authors present a physics experiment representing a practical session within a Physics course realized in collaboration by researchers of the University of Sannio, in Italy, and University of Thessaloniki in Greece.
\end{abstract}

Keywords: Web-based training, virtual laboratory, e-learning, light physics.

\section{INTRODUCTION}

The advent of Internet provided challenging new possibilities for teaching and learning. Especially the World Wide Web (www) makes it possible to develop and delivery educational contents in a whole new perspective.

In recent years, the development of online educational systems is on high priority for researchers, technologists, engineers and experts. The terms e-learning, web-based learning or hypermedia-aided learning are interchangeable for online education.

E-learning systems use various tools and technologies to establish its existence over World Wide Web. There are various standards and specifications developed so far. ADL (Advance Distributed Learning) SCORM (Sharable Content Object Reference Model) [2] is one of the most wellknown models for learning content objects. It provides interoperability between different learning systems by the means of standardized packaging, sequencing and communication methods for learning contents.

The major advantage of online learning is that it is accessible anytime and anywhere. User can access e-learning network anywhere in the world and take advantage of individualized learning. Online learning can be useful in industries to train employees and enhance their skill sets. It is useful in Universities for off-campus studies and it fulfills rapid training needs for all individuals who really want to utilize e-learning services. Beside these major advantages e-learning can provide facilities such as personalization, learning forums, digital libraries, virtual labs, authoring services, virtual classes and many others. Not only learners can take advantages of these services but teachers and authors can also find it useful in developing course contents or using it as a reference material.

In this framework the Remote Laboratory Distributed on Geographical Network LA.DI.RE. 
"G.Savastano" of University of Sannio was born, with the aim of developing a virtual learning environment to deliver, through the distance learning methodologies, theoretical lessons as well as laboratory activity in the Measurement and Instrumentation field. This new approach to the learning allows to reduce some drawbacks of handson laboratories as: (i) the high cost of measurement equipments and in general of the experimental laboratories in educational sites and industry, (ii) the growing number of students and technicians to be trained, (iii) the reduced number of laboratory technical staff, and (iv) the continuous evolution of measurement instrumentation involved, that makes it difficult and very expensive to keep the technical staff up-to-date.

The potentiality of remote teaching [3] and, in particular, the use of Internet as a channel to reach the students or workers at their homes was soon recognized [4,5]. Therefore, currently a lot of teaching material can be found as (i) Web based lectures and seminars [6,8], (ii) simulation of experiments $[9,10]$, and, more rarely, (iii) remotely accessible laboratories, where the learners can access real instrumentation through a Web page $[11,12]$.

The LA.DI.RE. "G.Savastano" system (http://www.misureremote.unisannio.it) presents a distributed platform based on a LMS allows providing full courses of electric and electronic measurement including theory as well as practical experiments on actual instrumentation. The LA.DI.RE. "G.Savastano" system integrates the advantages provided by an off-the-shelf LMS, compliant with international standards for web based training, and a new approach to provide remote experiments on measurement instrumentation.

In this paper the authors highlight the interdisciplinary aspect of the system that provides different theoretical and practical didactic materials about Electronics, Electric and Electronic Measurements, Automotive, Control Engineering, Biomedicine, Physics and so on. This innovative aspect makes this system unique and it has been possible thanking to the collaboration with several of Italian and International Universities. This paper underlines the interdisciplinary aspect showing a particular case as a real physics experiment that allows the students to verify the theoretical knowledge's acquired.

\section{LA.DI.RE. “G. SAVASTANO” SYSTEM}

LA.DI.RE. "G.Savastano" provides the students of electric and electronic measurements courses with the access to remote measurement laboratories delivering different didactic activities related to measurements experiments. With the introduction of this measurement laboratory a further step in Elearning evolution was introduced.

The initial infrastructure is composed of the laboratories of the University of Sannio and the University of Reggio Calabria "Mediterranea" under the patronage of the National Research Association on Electric and Electronic Measurement (GMEE) and the collaboration of about twenty Italian Universities and some specialized instrumentation, e-learning and publishing companies such as National Instruments, Tektronix, Agilent Technologies, Yokogawa, Keithley, Rockwell Automation, Didagroup, Augusta publishing.

The birth of the LA.DI.RE. "G.Savastano" system has promoted new collaborations between the University of Sannio and a lot of Universities of different countries as Brazil, Croatia, Czech Republic, Greece, Romania, Slovak Republic, Uruguay. In fact, at this moment on the system also courses in English, realized and introduced by researchers of these foreign Universities, are available and a lot of experiments of different scientific sectors, as measurement, biomedical, electronic, physical and automotive, are accessible. This aspect is very important because shows the interdisciplinary aspect of the LA.DI.RE. "G.Savastano" system, that represents an innovative platform for technology and know-how transfer and for the knowledge sharing including a remote laboratory for experiments on real instrumentation. This aspect allows a relevant knowledge sharing thanks to the distributed architecture of the system (Fig.1). Moreover the LA.DI.RE. "G.Savastano" system assures also a good socialization for the students and very realistic aspect. In fact the system provides some specific community services as virtual classroom and chat and especially the realtime visualization of instrumentation front panels thanks to IP cameras allowing the students to observe the instrument changes caused by their actions on the user interface [13].

\section{REMOTE EXPERIMENT IN OPTICAL PHYSICS}

In this section, after some description of the theoretical bases of the realized experiment, the bench set up and the control software will be sketched.

In order to understand the physical phenomenon that is examined with this experiment as well as the results that someone will have to expect from that, some of the basic aspects of the physic that studies the light, which is called optics, must be firstly stated. 


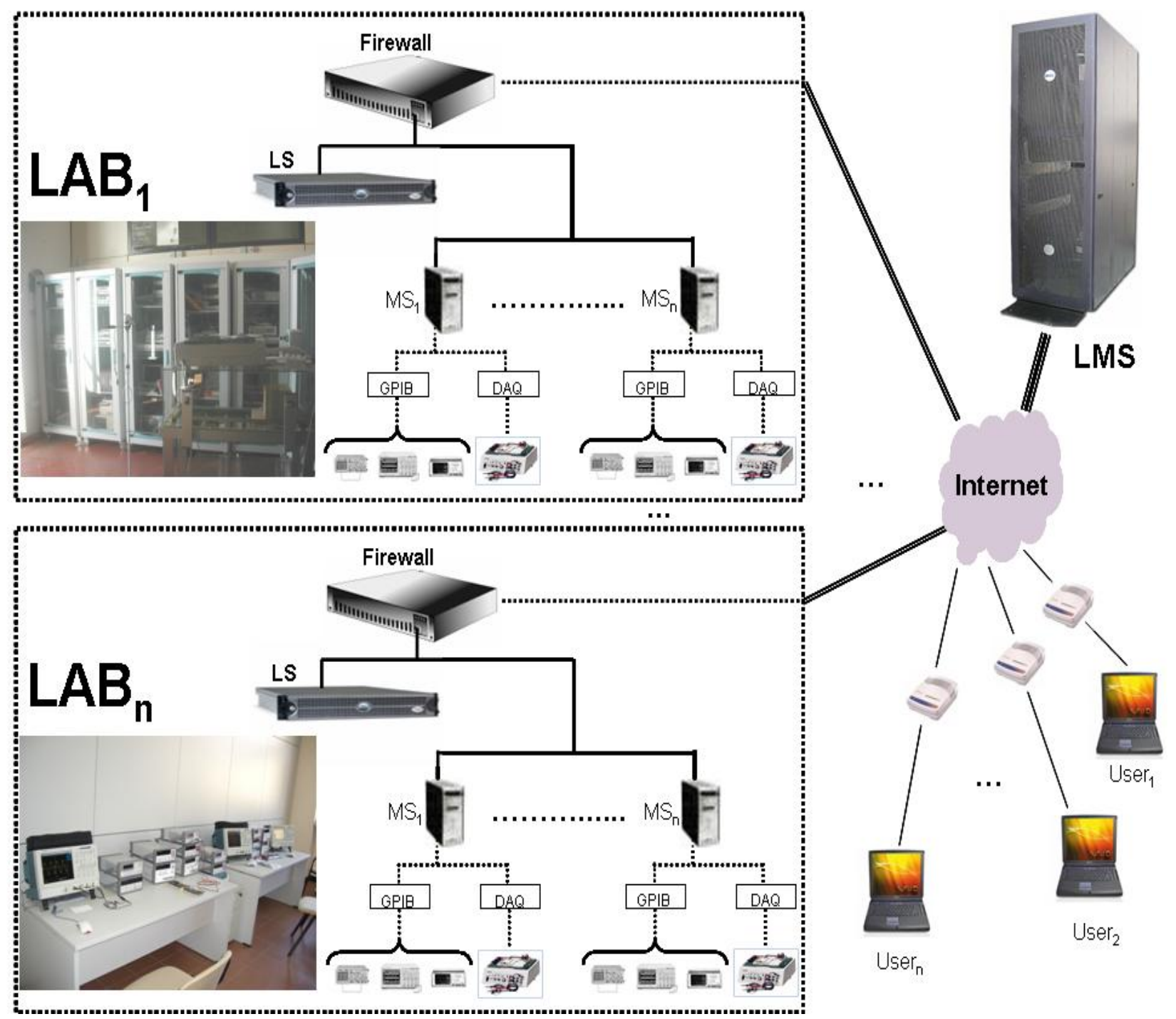

Fig. 1 - LA.DI.RE. G. Savastano architecture.

Looking back at the beginning of last century many researches have taken place and many new theories concerning the nature of the light have been proposed in order to explain a series of observed phenomena that could not be explained with the existed "classic" theory.

The nature of light seemed to confuse the scientists because some phenomena pointed that the light was consisted of participles while in some other phenomena was only explained with the acceptance of wave nature of the light. Many outstanding physicists worked on this problem and the results were the ignition for a new era in the scientific thought in general. After that the basics of most modern theories like electromagnetism, relativity and quantum mechanics were established. The results were in general lines that the nature of the light wasn't participle neither wave, but both.

Therefore the light could be supposed as many participles with zero mass when not moving or as an electromagnetic wave proportionally to the conditions and in this manner all the phenomena were fully explained. This double nature of the light is fully explained with the theory of quantum mechanics and it is not only characteristic of photons but of every participle moving with such great speed. Certainly the speed of the light is not any observed speed but the greater that can be achieved and this is one of the basic aspects of another theory, the famous theory of relativity.

As a conclusion we can treat the light as participles or as a wave in proportion with the kind of the phenomena that take place. Optics is the branch of physics that uses all the results on the nature of light to explain its properties and the phenomena that take place. Which are really many and with more applications.

Polarization of the light can be explained with the wave nature of the light. Light, as any electromagnetic wave, is composed of an electric and a magnetic field oscillating in orthogonal planes that are both orthogonal to the direction of the light propagation. The orientation of the intensity vector of the electric field, $E$ (and so of the magnetic one 
$H$ ) can vary with time and place. The variation of this orientation is generally coincidental, but in some cases this is not true. If this variation follows a specific low the light is called polarized. In particular the light is linearly polarized if the direction of the intensity vector of the electric field $E$ is constant. Generally the light is not polarized and it is called natural light. Polarized light can be produced through a variety of procedures. Of course polarized light cannot be detected by the human visual detector, the eye as only the direction of the intensity changes after a polarizer.

A device that produces linearly polarized light from natural one is called polarizer. The electric field direction is determined from the polarizer and is called the characteristic direction of the polarizer. In order to find the characteristic direction of a polarizer, another polarizer can be used as a detector. As it is possible to see in figure 2, after the first polarizer the light is polarized in a specific direction. After the second polarizer, which is called analyzer in such experiments, only the light component parallel to its characteristic direction pass through. Therefore, considering the electric field, only the component $E \cdot \cos \Phi$, with $\Phi$ the angle between the two characteristic directions, passes on the other side of the analyzer. The intensity of the light after the analyzer is arising to be given by the relationship:

$$
I=I_{0} \cdot \cos ^{2} \Phi
$$

which is known as Malus low and is the one that the remote student can study with the realized experiment [14].

Within the proposed experiment the user can control the relative angles of the two polarizers, observe and take measurements of the light coming from the analyzer through his personal computer, while the actual experiment is taking place at the same time in the LA.DI.RE. lab fully controlled from distance.

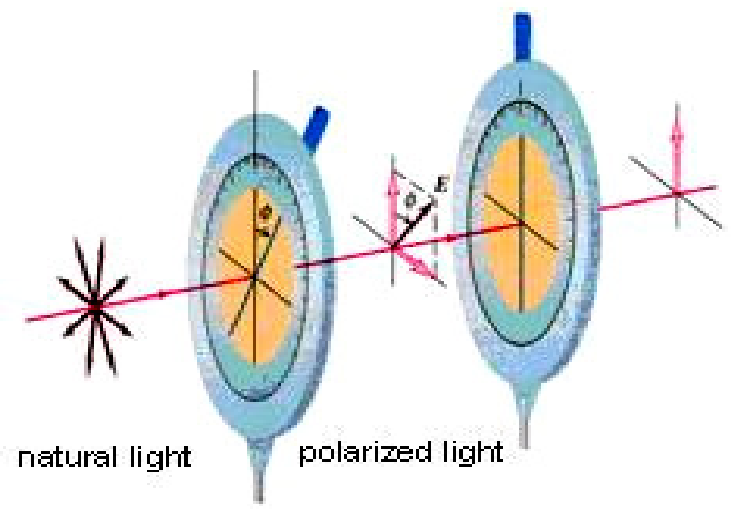

Fig. 2 - Analysis of polarized light.
This remote experiment on optics offers the opportunity to study the theory of the polarization of the light, its potential and its characteristics. It is possible to understand many elements concerning the nature of the light and the polarization, a phenomenon used in many modern applications.

Once this learning process is successfully completed, the remote user would have the ability of holding the experiment as if he had the experimental device in front of him and he can study the optical phenomenon of the light polarization. This allows the student to verify his theoretical knowledge regarding to light polarization through the remote control of the experiment.

\subsection{PHYSICS EXPERIMENT: HARDWARE COMPONENTS}

The actual experiment consists of a laser light source, two polarizers and a light sensor (from left to right in figure 3 ). The light source creates a straight, red laser beam that passes through the two polarizers to reach finally the light sensor.

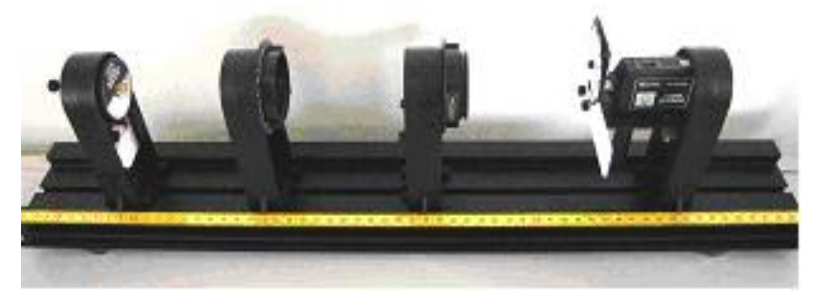

Fig. 3 - Experimental device for the study of polarization of the light.

Every known light source could have been used but the choice of this specific source was made because of the special advantages that a laser light offers. It has great intensity and directivity that help in the observation of the phenomenon. Each polarizer is placed on its base, which enables it to be rotated freely towards both directions. The plates of the two polarizers are parallel and both are vertical to the direction of the laser light beam that ends in the light detector and as a result. As a result every change of the intensity, due to change of the relative angle of the polarizers can be measured with the light sensor. In order to change the relative angle between the two polarizers, each of them can be rotated to any direction. An easy way to change and measure this angle is to start with the characteristic directions of the two polarizers aligned and then hold still the polarizer and start rotating the analyzer. The base of the analyzer is numbered in degrees and a stable spot on the base is used as pointer witch shows the angle of the rotation. The experiment actually includes the rotation of the analyzer, in a specific angle, which the user can change whenever he/she wants and measure the change on the 
intensity of the light beam. The use of a digital camera gives the opportunity also to watch the experiment happening, in real time.

These optical materials were all together ordered from the same provider (Pasco) and were easily mounted together and aligned. Up to this point the experimental devise is the traditional one that is used in every university optic laboratory for studying this phenomenon. The next task in the remote experiment that had to been made was the total control of the experimental devise through a computer. Firstly, the rotation of the analyzer has been obtained by means of a stepper motor that can be controlled from an electronic circuit or as in this experiment from a computer.

Stepper motor is ideal for this use as its rotation comes in small and precise steps. So that this rotation can be achieved it is necessary to drive currents inside the coils of the stepper with a special sequence. In order to do this we need a circuit that creates the appropriate sequence and another one for any coil responsible to drive the currents in each coil. The suitable and safe solution that has been adopted relies on the integrated circuit MC3479, a stepper motor driver, which makes all the required tasks to control the movement of the stepper.

As it can be seen in the connection diagram which is appeared in figure 4 , there are outputs of the integrated circuit (L1, L2, L3, L4) there are connected straight to the wires which lead to the coils of the stepper motor. The circuit needs only TTL control signals (inputs on the left part of the diagram) that bear all the information for the desirable movement of the stepper.

Voltage of Digital TTL signals can take values in a specific range which can be relatively between 2,5 and 5Volts when the logical signal has the "1" or "up" value, or between 0 and 2,5 Volts for " 0 " or "down" value. The control signals can have one of these values or, as for the case of clock signal, be a pulse that changes continually between these values with a specific frequency. Giving these signals to the circuit enables us to control the movement of the stepper. For instance when the control signal $\mathrm{CW} / \mathrm{CCW}$ has the value " 1 " the steppers rotor is rotating from left to write (counter clockwise). Of course for the movement to begin all the input had to take the right value and a clock pulse signal must be driven to the Clock input. Moreover one step occurs each time a clock pulse is coming to the circuit, so the frequency of the clock pulse determine the speed of the rotation.

The authors have used a small bipolar stepper motor mounted with the help of some plastic holds on the base of the polarizer as it appears in figure 5 . The motor motion is transferred to the analyzer ring with a plastic belt.

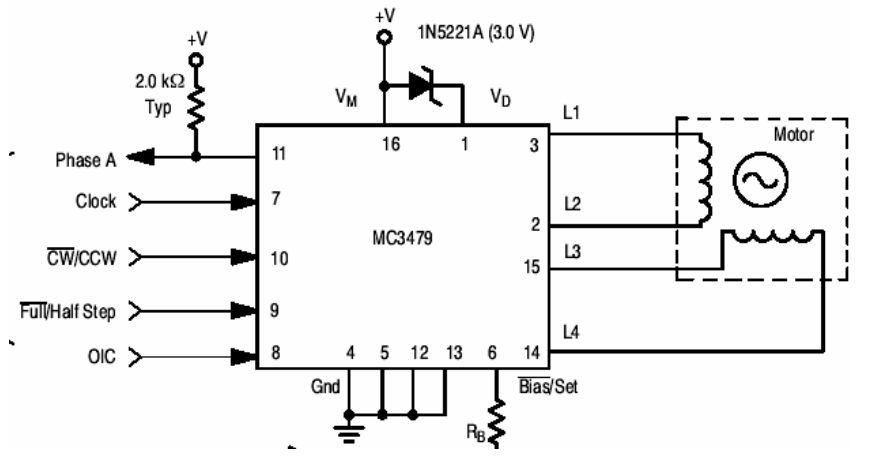

Fig. 4 - MC3479 control circuit.

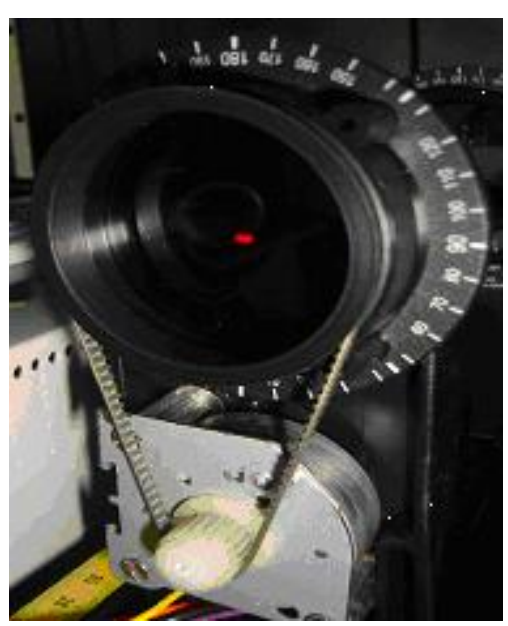

Fig. 5 - Final hardware device.

The theoretically required free rotation of the analyzer in any angle could be achieved, but not without errors. Due to mechanical restrictions, the rotation of the stepper could not be transferred correctly to the analyzer. This simple mechanical system was not able to work perfectly and as a result a number of steps, not predictable, were getting lost every time, because of some kick or slip of the belt on the analyzer. Possible solutions to this problem without a big increase in the cost of the bench are under examination.

The light sensor is general purpose, with high sensitivity. It has an analog output proportional to the intensity of the light that reaches it. This signal must reach the computer and should become available to the user. This task has been achieved with the use of the National Instruments "ELVIS". This is a multifunctional educational device, which is connected to the computer through a data acquisition board (DAQ) from the same producer and includes a breadboard where custom circuits can be built and connected to the DAQ. The motor control signals and the required power supplies have been obtained by building a control circuitry on the breadboard and connecting it to the digital outputs and the power supplies outputs of the DAQ board. 


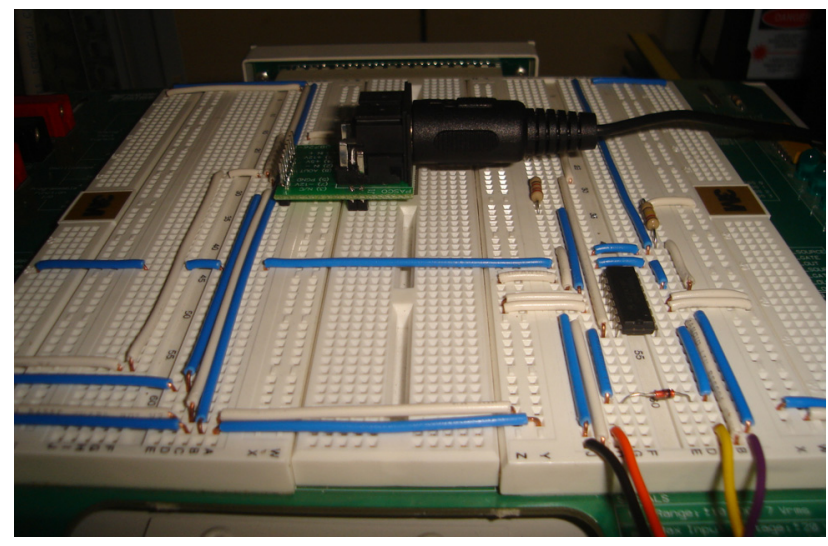

Fig. 6 - Electronic part for the control and the connection.

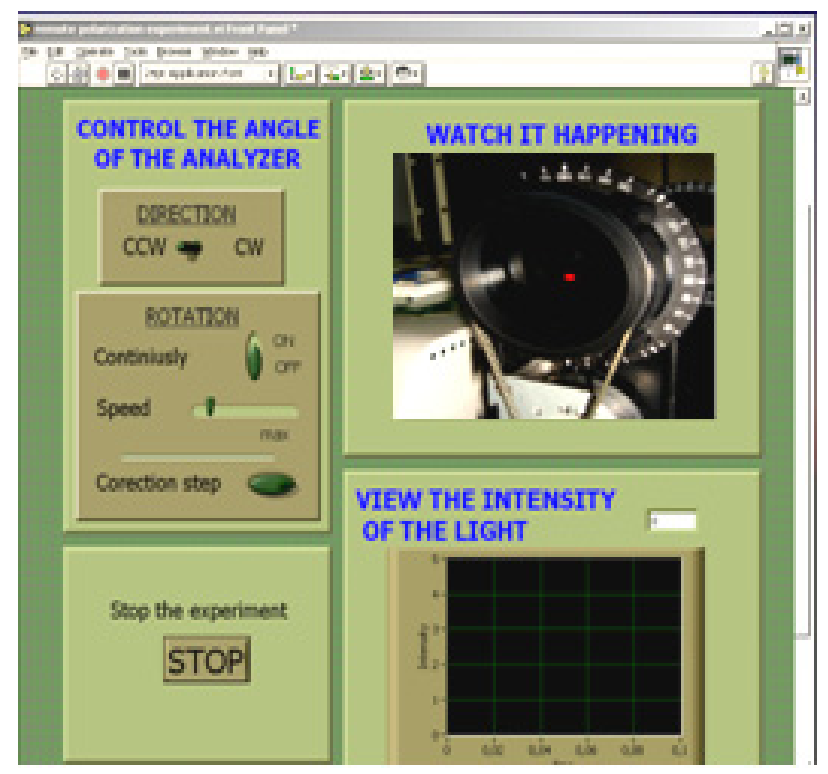

Fig. 7 - Software user interface.

On the same breadboard, through a bidirectional adapter it has been possible to provide the appropriate working voltages to the light sensor and to get its analog output that was guided to one analog input of the ELVIS. The final form of the breadboard with all the required control circuits and the adapter for the connection with the light sensor is appeared in figure 6.

Controlling the experiment now consists on programming the digital outputs of the DAQ and acquiring the analog output of the light sensor.

\subsection{PHYSICS EXPERIMENT: SOFTWARE USER INTERFACE}

The control software has been developed in the NI LabVIEW environment which includes a graphical programming language, developed by National Instruments especially for scientists and engineers. It offers great connectivity with measurement devices, a big number of special tools and great analysis capabilities.
Every program written in LabVIEW (or virtual instrument - VI, as it is called) is necessarily consisted of two parts, the Front panel and the Block diagram. In the window of the first one, the developer has the possibility to determine the way that the user interacts with the program. For this specific polarization experiment the front panel that was developed is the one that is presented in figure 6 . Through this graphical user interface, with the use of interactive buttons the user can control the rotation of the analyzer and watch the changes in the light intensity. There is also a window for video capturing of the rotary mechanical system. Every element of the front panel appears as a nod in the block diagram of the VI (figure 8). This is the part that the graphical programming is taking place. There is available every traditional programming operation but in the form of icons. While wiring the nods that represent the elements of the front panel, the data flows through the wires, and with contemporary use of graphical programming procedures the execution of the program is being accomplished.

The operation of the experiment is simple. On the front panel, there is a rotation switch, by putting this to the ON position, the analyzer rotates. The user is watching this rotation through the video window and also gets the value of the intensity on the relative display. When the angle is reaching the desirable value, the switch must return to the OFF position. There is also a button which gives a correction step each time it is pressed if there is a need to fix the angle value, another switch that determines the direction of the rotation and finally a STOP button, which stops the execution of the experiment when it is pressed.

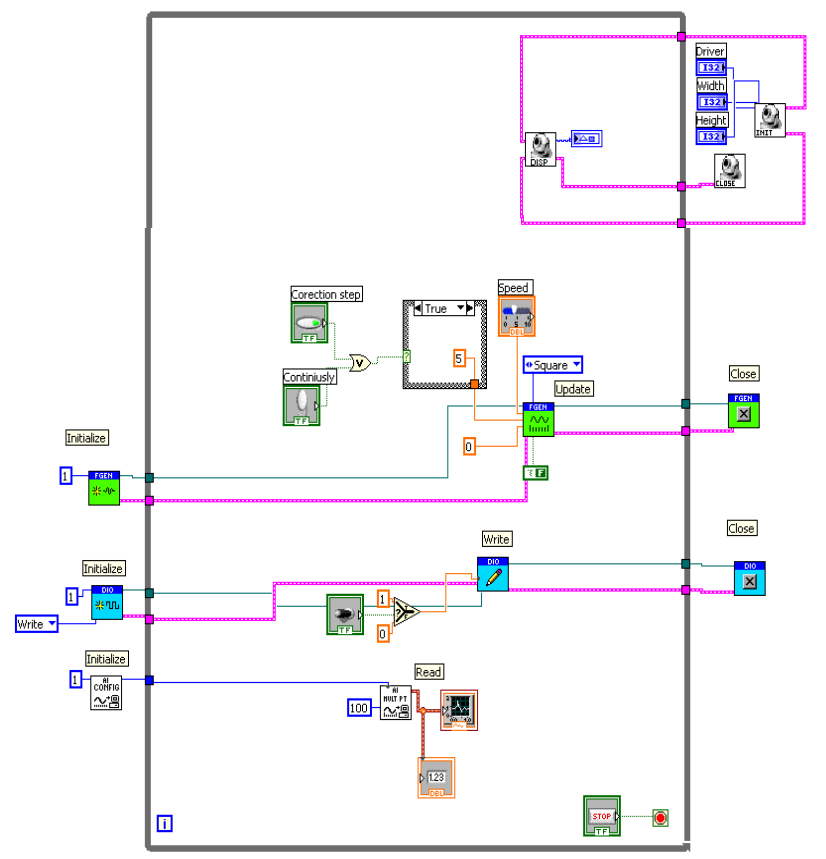

Fig. 8 - Block diagram of the VI. 
This approach is used because due to the previously mentioned mechanical inaccuracy it cannot be known, at each time the actual angle of the analyzer. It is necessary to remove the mechanical errors, or more simple to use a rotary motion sensor on the analyzer, so that we reach this. Moreover, if a more general view of the experiment is necessary, a second digital camera must be used.

\section{REFERENCES}

[1] S.Rapuano, F.Zoino, "A learning management system including laboratory experiments on measurement instrumentation", IEEE Trans. Instrumentation and Measurement, 55, 5, pp. 1757-1766.

[2] Advance Distributed Learning, Sharable Content Object Reference Model (SCORM 2004) Overview $2^{\text {nd }} \quad$ Edition, http://www.adlnet.org, July 2004.

[3] M.Cobby, D.Nicol, T.S.Durrani, W.A.Sandham, "Teaching electronic engineering via the World Wide Web", IEE Colloquium Computer Based Learning in Electronic Education, London, UK, 1995, pp. 7/1-11.

[4] G.C.Orsak, D.M.Etter, "Connecting the engineer to the 21 st Century through virtual teaching", IEEE Trans. on Education, vol.39, No.2, 1996, pp.165-172.

[5] M.A.Pine, L.A.Ostendorf, "Influencing earth system science education: NASA's approach", Int. Geoscience and Remote Sensing Symp., IGARSS'95, Florence, Italy, vol.I, 1995, pp.567-569.

[6] TechOnLine - Educational Resources for Electronics Engineers. http://www.techonline.com.

[7] NETwork per l'Università Ovunque. http://www.uninettuno.it .

[8] Laboratory of Signal Processing and Measurement Information - Engineering Faculty of University of Sannio. $\mathrm{http}: / /$ lesim1.ing.unisannio.it.

[9] Agilent Technologies Educator's Corner. http://www.educatorscorner.com/experiments/in dex.shtml.

[10] V.M.R. Penarrocha, M.F. Bataller, "Virtual instrumentation: first step towards a virtual laboratory", Proc. of IEEE Int. Workshop on virtual and intelligent measurement systems, Annapolis, Maryland (MD), USA, 2000, pp.5256.

[11] A.Bagnasco, A.M.Scapolla, "A grid of remote laboratory for teaching electronics", Proc. of 2nd Int. Learning Grid of Excellence $W G$ Workshop on e-Learning and Grid Technologies: a fundamental challenge for
Europe, $\quad$ Paris, 2003. http://ewic.bcs.org/conferences/2003/2ndlege/in dex.htm.

[12]L.Benetazzo, M.Bertocco, "A distributed training laboratory", Proc. of Eden Annual Conf. on Open and Distance Learning in Europe and Beyond, Granada, Spain, 2002, pp.409-414.

[13] N.Ranaldo, S.Rapuano, M.Riccio, F.Zoino “On the use of video-streaming technologies for remote monitoring of instrumentation", Proc. of IMTC-2006, Sorrento, Italy, 2006, pp.861-867.

[14]www.fisica.unige.it/ biologia/LABotticaMalus.pdf

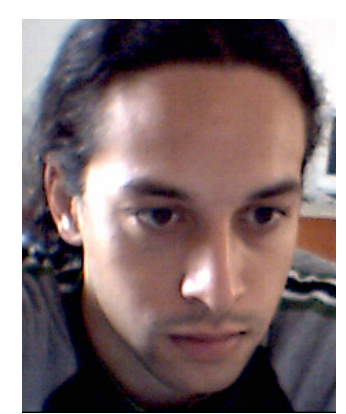

Antonios Apergis was born in Athens, on February of 1984. After finishing high school in the island of Tinos, he moved in Thessalonica to study physics on the Aristotle University of Thessalonica.

In the frameworks of Socrates - Erasmus programme, he spent the last semester of his studies in the University of Sannio in Benevento. There he joined the research on remote laboratories in LA.DI.RE, as a work for his dissertation thesis with had the subject "Development of a devise for the prosecuting of a real optics experiment on a distance laboratory".

In October of 2006 he received his degree on Physics. At the moment he is attending a master in Electronics and Telecommunications, in the physics department of Aristotle University.

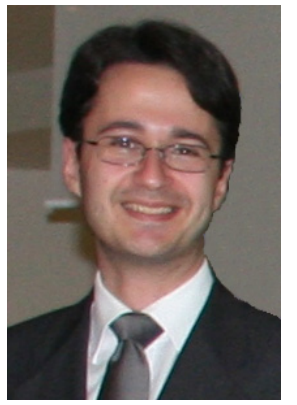

Sergio Rapuano received the master's degree (cum laude) in electronic engineering from the University of Salerno, Italy, in 1999 and the Ph.D. degree in computer science, telecommunications and applied electromagnetism from the same University in 2003.

In 1999 he joined the research activities carried out at the Laboratory of Signal Processing and Measurement Information of the University of Sannio, Benevento. In 2002 he joined the Faculty of Engineering of the University of Sannio as Assistant Professor of Electric and Electronic Measurement.

$\mathrm{He}$ is currently developing his research work in digital processing for measurement in telecommunications, data converters, distributed measurement systems and virtual laboratories, medical measurement.

Sergio Rapuano is a member of the IEEE Instrumentation and Measurement Society TC-10 and Secretary of the TC-23 Working Group on "etools for Education in Instrumentation and Measurement". 


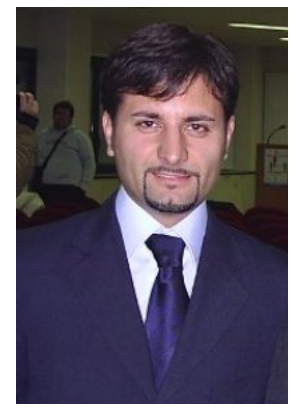

Francesco Zoino was born in

Benevento, on August 8, 1976.

In 2004 he achieved the master's degree in Software

Engineering from University of

Sannio presenting a thesis work titled "A new method for the detection of harmonics in the Power Quality".

At the present time he is a PhD Student in Software Engineering and works with the Laboratory of Signal Processing and Measurement Information of the University of Sannio, Benevento. In particular his research work deals with the remote control of measurement instrumentation over the Internet and the medical measurement applications. 